\title{
Comparative evaluation of the effects of platelet-rich plasma formulations on extracellular matrix formation and the NF-кB signaling pathway in human articular chondrocytes
}

\author{
WENJING YIN*, HAITAO XU*, JIAGEN SHENG, ZHENGLIANG XU, XUETAO XIE and CHANGQING ZHANG
}

\author{
Department of Orthopaedic Surgery, Shanghai Jiao Tong University \\ Affiliated Sixth People's Hospital, Shanghai 200233, P.R. China
}

Received January 26, 2016; Accepted January 16, 2017

DOI: $10.3892 / \mathrm{mmr} .2017 .6365$

\begin{abstract}
Concentrated leukocytes in leukocyte and platelet-rich plasma (L-PRP) may deliver increased levels of pro-inflammatory cytokines to activate the nuclear factor (NF)- $\mathrm{kB}$ signaling pathway, to counter or overwhelm the beneficial effects of growth factors on cartilage regeneration. However, to date, no relevant studies have substantiated this. In the present study, L-PRP and pure platelet-rich plasma (P-PRP) were prepared, and leukocytes, platelets, pro-inflammatory cytokines and growth factor concentrations were quantified; they were then used to treat human articular chondrocytes (HACs). Pyrrolidine dithiocarbamate (PDTC; $50 \mu \mathrm{M}$ ) was used to inhibit the activation of NF- $\mathrm{KB}$. The nuclear translocation of NF- $\mathrm{kB}$ p 65 and the protein expression of cartilaginous markers (collagen II, aggrecan and sex-determining region Y-box 9) were determined using western blot analysis. The mRNA expression of NF- $\mathrm{KB}$-dependent inflammatory mediators, including inducible nitric oxide synthase and cyclooxygenase- 2 , and cartilaginous markers were determined using reverse transcription-quantitative polymerase chain reaction analysis. The production of prostaglandin E2, nitric oxide and glycosaminoglycan (GAG) were quantified using enzyme-linked immunosorbent assays, the Griess reaction and a 1,9-dimethylmethylene blue assay, respectively. The results demonstrated that L-PRP induced the nuclear translocation of NF-kB p65, upregulated the mRNA expression of
\end{abstract}

Correspondence to: Professor Changqing Zhang or Dr Xuetao Xie, Department of Orthopaedic Surgery, Shanghai Jiao Tong University Affiliated Sixth People's Hospital, 600 Yishan Road, Shanghai 200233, P.R. China

E-mail: zhangcq@sjtu.edu.cn

E-mail: xuetaoxie@163.com

*Contributed equally

Key words: platelet-rich plasma, leukocytes, cytokines, nuclear factor- $\kappa \mathrm{B}$, extracellular matrix formation, human articular chondrocytes
NF- $\mathrm{BB}$-dependent inflammatory mediators and upregulated the production of their products, whereas P-PRP, which had similar growth factor concentrations but significantly lower pro-inflammatory cytokine concentrations than L-PRP, did not. P-PRP promoted the mRNA and protein expression levels of cartilaginous markers and the production of GAG more effectively, compared with L-PRP. Furthermore, inhibition of the activation of NF- $\mathrm{KB}$ by PDTC enhanced the effects of L-PRP on extracellular matrix formation in the HACs to a level similar to that of P-PRP. These findings suggested that leukocytes in L-PRP activated the NF-kB signaling pathway via the delivery of interleukin- $1 \beta$ and tumor necrosis factor- $\alpha$ to counter the beneficial effects of growth factors on extracellular matrix formation in HACs. Therefore, P-PRP may be more suitable for the treatment of osteoarthritis.

\section{Introduction}

Osteoarthritis, which is characterized by the destruction of articular cartilage and leads to pain and loss of function, primarily in the knees and hips, is one of the most common sources of physical disability resulting in impaired quality of life (1). Numerous clinical strategies, including the oral administration of nonsteroidal anti-inflammatory drugs (2) and intra-articular injection of hyaluronan (3), have been used to prevent the progression of osteoarthritis. Despite providing pain relief, these therapies offer limited improvement in cartilage regeneration due to the minimal blood supply, limited extracellular matrix formation and low cell density of the tissue (4).

Platelet-rich plasma (PRP) is an autologous blood product, which contains concentrated platelets. The $\alpha$-granules of concentrated platelets in PRP contain and release concentrated levels of growth factors, which promote chondrocyte proliferation and cartilaginous matrix secretion, including platelet-derived growth factor (PDGF), transforming growth factor (TGF), insulin-like growth factor (IGF), fibroblast growth factor (FGF) and epidermal growth factor (EGF) $(5,6)$. Consequently, PRP has increased in popularity over the last decade for the treatment of osteoarthritis, functioning as a growth factor delivery medium to assist in cartilage regeneration $(7,8)$. 
Due to the increasing use of PRP in clinical practice, various protocols for PRP preparations are available, and different protocols result in PRP formulations differing in cellular composition, particularly leukocyte concentration (9). It has been demonstrated that leukocyte and platelet-rich plasma (L-PRP), which has a high leukocyte concentration, also has high concentrations of pro-inflammatory cytokines, including interleukin (IL)-1 $\beta$ and tumor necrosis factor (TNF)- $\alpha$, whereas pure platelet-rich plasma (P-PRP), which concentrates platelets and depletes leukocytes, has low or undetectable pro-inflammatory cytokine concentrations (10). The stimulation of articular chondrocytes by IL- $1 \beta$ or TNF- $\alpha$ has been described to allow the nuclear translocation of $\mathrm{NF}-\kappa \mathrm{B}$ p 65 , which then activates the expression of a wide range of catabolic genes, including inducible nitric oxide synthase (iNOS) and cyclooxygenase-2 (COX-2) in chondrocytes, resulting in the production of destructive proteases and enhanced degradation of extracellular matrix $(11,12)$. Therefore, the high levels of IL-1 $\beta$ and TNF- $\alpha$ in L-PRP may activate the NF- $\kappa B$ signaling pathway to induce harmful effects on cartilage regeneration, to counter or overwhelm the beneficial effects of growth factors. However, to date, no relevant studies have substantiated this.

The aims of the present study were to evaluate the effects of L-PRP and P-PRP on extracellular matrix formation, and the $\mathrm{NF}-\kappa \mathrm{B}$ signaling pathway in human articular chondrocytes (HACs) in vitro.

\section{Materials and methods}

Participant recruitment. The present study was performed in accordance with the principles of the Declaration of Helsinki. The Independent Ethics Committee of Shanghai Jiao Tong University Affiliated Sixth People's Hospital (Shanghai, China) approved the protocols of the study. Written informed consent was obtained from each volunteer.

A total of eight patients (six men and two women; 48-60 years old) with knee osteoarthritis were recruited for the donation of articular cartilage samples between January and February 2015. The diagnosis of knee osteoarthritis was based on the American College of Rheumatology criteria for symptomatic primary knee osteoarthritis (13). In addition, 10 healthy volunteers (seven men and three women; 26-54 years old) were recruited for blood donation between February and March 2015. The inclusion criteria for this group consisted of healthy adults with no medical history of relevant diseases or consumption of any medications known to affect platelet function or concentration for 21 days prior to study commencement.

PRP preparation. Venous blood $(\sim 63 \mathrm{ml})$ was collected from each blood donor and anticoagulated with $7 \mathrm{ml}$ acid-citrate dextrose solution A. Of the anticoagulated blood obtained, $\sim 68 \mathrm{ml}$ was used for L-PRP and P-PRP preparation in a single-donor model, $0.5 \mathrm{ml}$ was used for whole blood analysis, and $1.5 \mathrm{ml}$ was used for the quantification of growth factor and pro-inflammatory cytokine concentrations.

The WEGO PRP preparation system (WEGO, Shandong, China), the only commercial PRP preparation system approved by the Chinese Food and Drug Administration, was used for L-PRP preparation, according to the manufacturer's protocol. In brief, $28 \mathrm{ml}$ of anticoagulated blood was spun at $400 \mathrm{x} g$ for $10 \mathrm{~min}$ at room temperature in a specific centrifuge. Following the first spin, the blood was separated into three components: Erythrocytes at the bottom, buffy coat in the middle and platelet-containing plasma at the top. The majority of the erythrocytes were eliminated, and the resulting buffy coat and platelet-containing plasma were spun again for $10 \mathrm{~min}$ at $400 \mathrm{x} \mathrm{g}$ at room temperature. Following removal of the platelet-poor plasma supernatant, the precipitated platelets were resuspended in the residual plasma to obtain $4 \mathrm{ml}$ of L-PRP.

P-PRP was prepared using a method developed in the laboratory at Shanghai Jiao Tong University Affiliated Sixth People's Hospital, which was demonstrated to concentrate platelets in a similar manner to the WEGO PRP preparation system, but removing the majority of leukocytes and erythrocytes (data not shown). To prepare P-PRP using this method, a $40 \mathrm{ml}$ volume of the anticoagulated whole blood was spun at $160 \mathrm{x} g$ for $10 \mathrm{~min}$ at room temperature in a $50-\mathrm{ml}$ centrifuge tube to separate the platelet-containing plasma from the erythrocytes and buffy coat, following which the platelet-containing plasma was collected by gentle aspiration, transferred to a new tube and spun again at $250 \mathrm{x} g$ for $15 \mathrm{~min}$ at room temperature. The platelet-poor plasma supernatant was discarded and the precipitated platelets were resuspended in the residual plasma to obtain $4 \mathrm{ml}$ of P-PRP.

Quantification of leukocyte and platelet concentrations. The leukocyte and platelet concentrations of the whole blood, L-PRP and P-PRP were determined via whole blood analysis using an automatic hematology analyzer (XS-800i; Sysmex Corporation, Kobe, Japan) in the clinical laboratory of Shanghai Jiao Tong University Affiliated Sixth People's Hospital.

Quantification of growth factors and pro-inflammatory cytokine concentrations. The concentrations of PDGF-AB, TGF- $\beta 1$, IL- $1 \beta$ and TNF- $\alpha$ in the whole blood, L-PRP and P-PRP samples were quantified according to the protocols described previously (14). In brief, the whole blood, L-PRP and P-PRP samples were incubated with $10 \% \mathrm{CaCl}_{2}$ (final concentration, $22.8 \mathrm{mM}$ ) at $37^{\circ} \mathrm{C}$ in a humidified atmosphere containing $5 \% \mathrm{CO}_{2}$ for 7 days. At the end of the incubation period, the supernatants were collected, and the concentrations of growth factors and pro-inflammatory cytokines were determined using an enzyme-linked immunosorbent assay (ELISA) with the Quantikine Human Immunoassay kits (R\&D Systems, Inc., Minneapolis, MN, USA) according to manufacturer's protocol.

Isolation and treatment of HACs. The HACs were isolated according to the protocols reported previously (15). In brief, articular cartilage samples were obtained from patients undergoing total knee replacement surgery, minced into small pieces and digested using $0.1 \%$ collagenase II (Sigma-Aldrich; Merck Millipore, Darmstadt, Germany) in Dulbecco's modified Eagle's medium/Ham's F-12 50/50 mix (DMEM/F12; Corning Life Sciences, Lowell, MA, USA). The released HACs were cultured in DMEM/F12 containing 10\% fetal bovine serum (FBS; Gibco; Thermo Fisher Scientific, Inc., Waltham, MA, USA) and $1 \%$ antibiotics $(10,000 \mathrm{U} / \mathrm{ml}$ penicillin and 
$10,000 \mu \mathrm{g} / \mathrm{ml}$ streptomycin; Gibco; Thermo Fisher Scientific, Inc, ) at $37^{\circ} \mathrm{C}$ in a humidified atmosphere containing $5 \% \mathrm{CO}_{2}$. The medium was replaced every 2 days. The cells were passaged at $\sim 90 \%$ confluence.

HACs at the third passage were seeded a density of $2.5 \times 10^{5} /$ well in the lower chambers of 6 -well Transwell systems (Corning Life Sciences) and divided into four groups: i) Control group, comprising cells incubated with serum-free DMEM/F12 for $24 \mathrm{~h}$ and then treated with DMEM/F12 supplemented with $10 \%$ FBS; ii) L-PRP group, comprising serum-starved cells, as above, treated with DMEM/F12 containing 10\% L-PRP; iii) PDTC group, comprising cells serum-starved with serum-free DMEM/F12 containing $50 \mu \mathrm{M}$ PDTC (Sigma-Aldrich; Merck Millipore) for $24 \mathrm{~h}$ prior to L-PRP treatment; iv) P-PRP group, comprising cells serum-starved with DMEM/F12 for $24 \mathrm{~h}$ and then treated with DMEM/F12 supplemented with 10\% P-PRP.

PDTC is an inhibitor of the NF- $\mathrm{KB}$ signaling pathway and $50 \mu \mathrm{M}$ PDTC has been reported to inhibit NF- $\kappa \mathrm{B}$ activation in multiple cell lines $(16,17)$. The most frequently used PRP concentration in in vitro studies is $10 \%$, and may be comparable to the concentration of PRP reached during in vivo administration (18-20). The PRP formulations were placed in the upper chambers of Transwell systems and activated with $10 \% \mathrm{CaCl}_{2}$ (final concentration, $22.8 \mathrm{mM}$ ) to avoid direct contact between PRP clots and HACs, which were seeded in the lower chambers (Fig. 1) (14).

Sample collection. The cells were collected following $24 \mathrm{~h}$ of FBS, L-PRP or P-PRP treatment for the analysis of NF- $\mathrm{KB}$ p65 translocation, at $96 \mathrm{~h}$ for reverse transcription-quantitative polymerase chain reaction (RT-qPCR) analysis and at 7 days for western blot analysis. For analyzing the production of prostaglandin E2 (PGE2), nitric oxide (NO) and glycosaminoglycan (GAG) by HACs, the medium was replaced with serum-free medium following treatment with FBS, L-PRP or P-PRP for 7 days to minimize potential artifacts due to the presence of PGE2, NO and GAG in the blood products. After $24 \mathrm{~h}$, the conditioned media were collected for the analyses.

Western blot analysis. The expression of NF-кB p65 in the nuclei of HACs was analyzed using western blot analysis. The nuclear protein extracts were prepared using an NE-PER Nuclear and Cytoplasmic Extraction Reagents kit (Thermo Fisher Scientific, Inc.) according to manufacturer's protocol. The total protein concentration was detected using a BCA Protein Assay kit (Pierce; Thermo Fisher Scientific, Inc.). Proteins $(50 \mu \mathrm{g})$ were separated by $10 \%$ SDS-PAGE and subsequently transferred onto a PVDF membrane. The membranes were blocked with a solution of low-fat milk, washed three times for 5 min each with Tris-buffered saline with Tween 20 (TBST), and subsequently incubated with anti-NF-kB p65 (catalog no. 8242; Cell Signaling Technology, Inc., Danvers, MA, USA; 1:1,000) or anti-GAPDH (catalog no. 5174, Cell Signaling Technology, Inc.; 1:1,000) at $4^{\circ} \mathrm{C}$ overnight. Following three washes for 5 min each with TBST, the membranes were incubated with a horseradish peroxidase-conjugated anti-rabbit IgG secondary antibody (catalog no. 7074; Cell Signaling Technology, Inc.; $1: 1,000$ ) at $37^{\circ} \mathrm{C}$ for $1 \mathrm{~h}$, followed by a further three washes for $5 \mathrm{~min}$ each with
TBST. The blots were subjected to chemiluminescence detection using ECL western blotting substrate (Pierce; Thermo Fisher Scientific, Inc.).

The protein expression levels of cartilaginous markers, including collagen II (Col II), aggrecan and sex-determining region Y-box 9 (SOX9), in the cultured HACs were also analyzed using western blot analysis. The cells were lysed with mammalian protein extraction reagent (Pierce; Thermo Fisher Scientific, Inc.) supplemented with complete protease inhibitor. Western blot analysis was then performed, as above, using antibodies against Col II (catalog no. ab34712; Abcam, Cambridge, MA, USA; 1:5,000), aggrecan (catalog no. ab53731; Abcam; 1:500), SOX9 (catalog no. ab26414; Abcam; 1:1,000), anti-GAPDH, and anti-rabbit IgG.

RT-qPCR analysis. RT-qPCR analysis was performed to detect the mRNA expression levels of cartilaginous markers (Col II, aggrecan and SOX9) and NF- $\mathrm{kB}$-dependent inflammatory mediators (iNOS and COX-2). Total RNA was extracted from the cells using TRIzol reagent (Invitrogen; Thermo Fisher Scientific, Inc.), and the quantity and purity were measured using a NanoDrop 2000 spectrophotometer (Thermo Fisher Scientific, Inc.). The RT reaction was performed using EasyScript one-step gDNA Removal and cDNA Synthesis Supermix (TransGen Biotech Co., Ltd., Beijing, China) from $1 \mu \mathrm{g}$ of total RNA, according to the manufacturer's protocol. The qPCR analysis was performed using TransStart Tip Green qPCR SuperMix (TransGen Biotech Co., Ltd.). The total volume of the reaction system was $10 \mu \mathrm{l}$, including $1 \mu \mathrm{l} \mathrm{cDNA}, 0.2 \mu \mathrm{l}$ forward primer $(10 \mu \mathrm{mol} / \mathrm{l}), 0.2 \mu \mathrm{l}$ reverse primer $(10 \mu \mathrm{mol} / \mathrm{l}), 0.2 \mu \mathrm{l}$ 50X passive reference dye, $3.4 \mu \mathrm{l}$ double-distilled water and $5 \mu 1$ Tip Green qPCR SuperMix. The reaction conditions were as follows: 40 cycles of initiation at $95^{\circ} \mathrm{C}$ for $30 \mathrm{sec}$, annealing at $95^{\circ} \mathrm{C}$ for $5 \mathrm{sec}$, and extension at $60^{\circ} \mathrm{C}$ for $30 \mathrm{sec}$. GAPDH was used as a housekeeping gene for the normalization of data. The primer sequences for the target genes are listed in Table I. Data were analyzed using the $2^{-\Delta \Delta \mathrm{Cq}}$ method, as described previously (21).

Analysis of the production of PGE2,NO and GAG. The concentrations of the products of iNOS and COX-2 (NO and PGE2, respectively) and GAG in the conditioned medium were quantified via ELISA with a Quantikine Human Immunoassay kit (R\&D Systems, Inc.), the Griess reaction using an NO Assay kit (Thermo Fisher Scientific, Inc.) and a 1,9-dimethylmethylene blue assay using a GAG assay kit (Biocolor, Carrickfergus, UK), respectively, according to manufacturers' protocols. The data were calculated as fold changes compared with the control group.

Statistical analysis. Data were collected from triplicate parallel samples and were analyzed using the Statistical Package for Social Sciences version 22.0 (IBM SPSS, Armonk, NY, USA) and presented as the mean \pm standard deviation. One-way analysis of variance and the Bonferroni post-hoc test were performed to analyze the differences between groups. Pearson correlation analysis was used to analyze the linear correlations between cytokine concentrations and platelet concentrations, and the leukocyte concentrations of PRP formulations. $\mathrm{P}<0.05$ was considered to indicate a statistically significant difference. 
Table I. Primers sequences used for quantitative-polymerase chain reaction analysis.

\begin{tabular}{lll}
\hline Gene & Forward primer sequence $\left(5^{\prime}-3^{\prime}\right)$ & Reverse primer sequence $\left(5^{\prime}-3^{\prime}\right)$ \\
\hline Col II & GACAATCTGGCTCCCAAC & ACAGTCTTGCCCCACTTAC \\
Aggrecan & TCGAGGACAGCGAGGCC & TCGAGGGTGTAGCGTGTAGAGA \\
SOX9 & AGCTCACCAGACCCTGAGAA & TCCCAGCAATCGTTACCTTC \\
COX-2 & CTTCACGCATCAGTTTTTCAAG & TCACCGTAAATATGATTTAAGTCCAC \\
iNOS & GCTGCCAAGCTGAAATTGA & GATAGCGCTTCTGGCTCTTG \\
GAPDH & ATCCCATCACCATCTTCC & GAGTCCTTCCACGATACCA
\end{tabular}

Col II, collagen II; SOX9, sex-determining region Y-box 9; iNOS, inducible nitric oxide synthase; COX-2, cyclooxygenase-2.

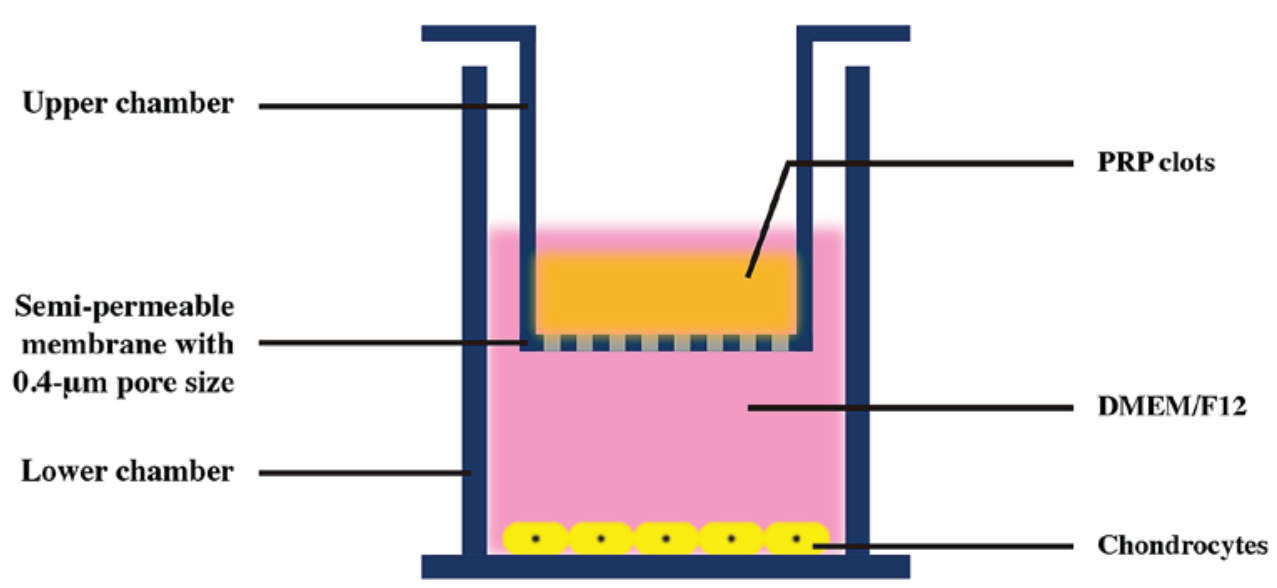

Figure 1. Transwell system used in the present study.

\section{Results}

Components of whole blood, L-PRP and P-PRP samples. The mean leukocyte, IL-1 $\beta$ and TNF- $\alpha$ concentrations in the whole blood samples were $6.28 \pm 1.60 \times 10^{9} / 1,7.70 \pm 4.85$ and $4.54 \pm 2.33 \mathrm{pg} / \mathrm{ml}$, respectively. Compared with the whole blood, the concentrations of leukocytes in the L-PRP samples were significantly higher $\left(36.37 \pm 9.67 \times 10^{9} / 1 ; \mathrm{P}<0.001 ;\right.$ Fig. $\left.2 \mathrm{~A}\right)$, as were those of IL-1 $\beta(127.32 \pm 68.07 \mathrm{pg} / \mathrm{ml} ; \mathrm{P}<0.001$; Fig. 2B) and TNF- $\alpha$ (53.07 $\pm 34.19 \mathrm{pg} / \mathrm{ml}$; P<0.001; Fig. 2C). Although P-PRP had significantly lower concentrations of leukocytes, IL-1 $\beta$ and TNF- $\alpha\left(0.12 \pm 0.12 \times 10^{9} / 1,3.85 \pm 2.43\right.$ and $2.27 \pm 1.17 \mathrm{pg} / \mathrm{ml}$, respectively), compared with L-PRP $(\mathrm{P}<0.001)$, the differences in concentrations between P-PRP and whole blood were not significant $(\mathrm{P}=0.114 ; \mathrm{P}>0.999$ and $\mathrm{P}>0.999$, respectively).

The mean platelet, PDGF-AB and TGF- $\beta 1$ concentrations in the whole blood were $218.47 \pm 43.09 \times 10^{9} / 1,5.62 \pm 1.88$ and $17.26 \pm 5.23 \mathrm{ng} / \mathrm{ml}$, respectively. Compared with the whole blood, the L-PRP and P-PRP showed increased concentrations of platelets $\left(1,202.00 \pm 369.96\right.$ and $1,258.30 \pm 224.16 \times 10^{9} / 1$, respectively; Fig. 2D), PDGF-AB (34.21 \pm 9.26 and $35.51 \pm 11.27 \mathrm{ng} / \mathrm{ml}$, respectively; Fig. 2E) and TGF- $\beta 1$ $(98.75 \pm 29.78$ and $103.58 \pm 31.41 \mathrm{ng} / \mathrm{ml}$, respectively; Fig. $2 \mathrm{~F})$. No significant differences in concentrations of platelets, PDGF-AB or TGF- $\beta 1$ were found between L-PRP and P-PRP ( $P>0.999$ and $P>0.999$ and $P>0.999$, respectively).
The results of the correlation analysis are shown in Fig. 3. There were significant positive correlations between the leukocyte concentrations and the IL- $1 \beta$ concentration $(r=0.924$; $\mathrm{P}<0.001$; Fig. 3A) and TNF- $\alpha$ concentration of the PRP formulations ( $r=0.892$; $P<0.001$; Fig. 3B). Significant positive correlations were also observed between the platelet concentration and the PDGF-AB ( $\mathrm{r}=0.760 ; \mathrm{P}<0.001$; Fig. $3 \mathrm{C})$ and TGF- $\beta 1$ concentrations of PRP formulations ( $r=0.812$; $\mathrm{P}<0.001$; Fig. 3D). However, no significant correlations were found between the leukocyte concentrations and the PDGF-AB ( $r=0.028 ; \mathrm{P}=0.908$; Fig. 3E) and TGF- $\beta 1$ concentrations of PRP formulations $(\mathrm{r}=0.035 ; \mathrm{P}=0.882$; Fig. $3 \mathrm{~F})$, or between platelet concentrations and the IL-1 $\beta$ ( $\mathrm{r}=0.108$; $\mathrm{P}=0.650$; Fig. $3 \mathrm{G})$ and TNF- $\alpha$ concentrations of PRP formulations ( $\mathrm{r}=0.225 ; \mathrm{P}=0.340$; Fig. $3 \mathrm{H})$.

Effects of L-PRP and P-PRP on the $N F-\kappa B$ signaling pathway. Western blot analysis showed that P-PRP did not affect the translocation of NF- $\kappa \mathrm{B}$ p65, whereas L-PRP markedly induced the accumulation of NF- $\kappa \mathrm{B}$ p65 in the nuclei of HACs, which was reversed by PDTC (Fig. 4A). In addition, the HACs in the L-PRP group demonstrated upregulated mRNA expression levels of COX-2 ( $\mathrm{P}<0.001$; Fig. 4B) and iNOS $(\mathrm{P}<0.001$; Fig. 4C), and enhanced production of PGE2 (P<0.001; Fig. 4D) and NO ( $\mathrm{P}<0.001$; Fig. 4E), compared the control group and P-PRP group. PDTC significantly inhibited these effects of L-PRP $(\mathrm{P}<0.001)$. No significant differences in the mRNA expression of COX-2 or iNOS or the production of PGE2 or 
A
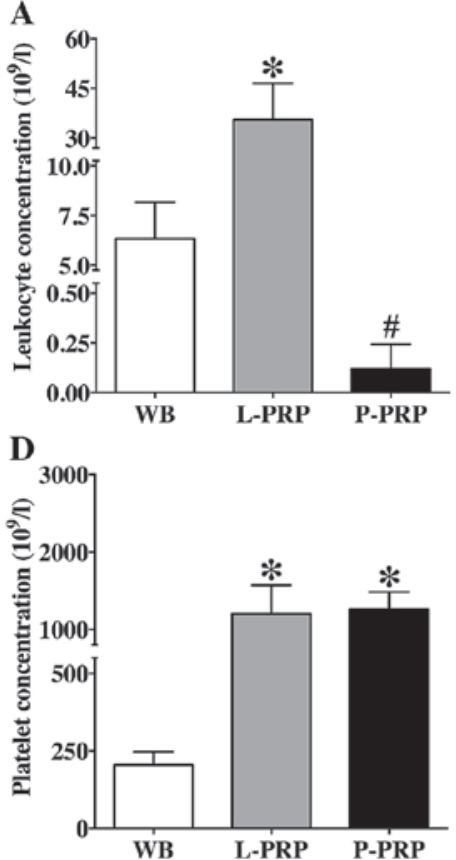

B
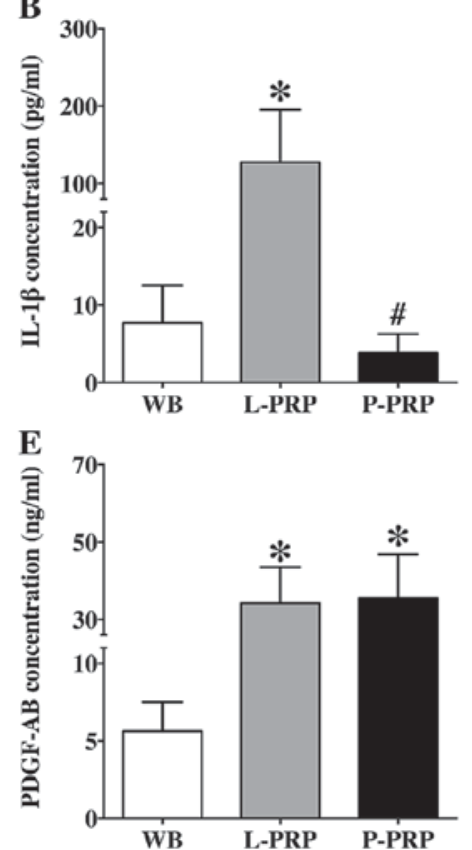

C
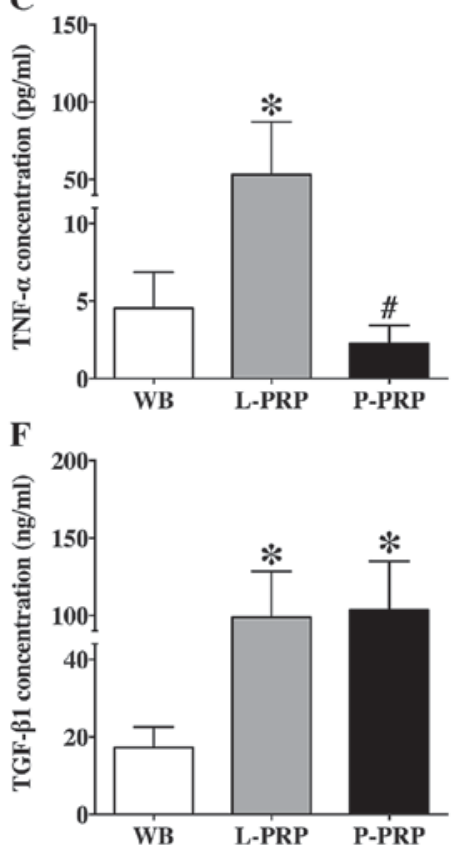

Figure 2. Concentrations of (A) leukocytes, (B) IL-1 $\beta$, (C) TNF- $\alpha$, (D) platelets, (E) PDGF-AB and (F) TGF- $\beta 1$ of WB and PRP formulations. Data are presented as the means \pm standard deviation. ${ }^{*} \mathrm{P}<0.05$ between WB and PRP formulations; ${ }^{*} \mathrm{P}<0.05$ between L-PRP and P-PRP. WB, whole blood; L-PRP, leukocyte and platelet-rich plasma; P-PRP, pure platelet-rich plasma; IL-1 $\beta$, interleukin-1 $\beta$; TNF- $\alpha$, tumor necrosis factor- $\alpha$; PDGF-AB, platelet-derived growth factor-AB; TGF- $\beta 1$, transforming growth factor- $\beta 1$.
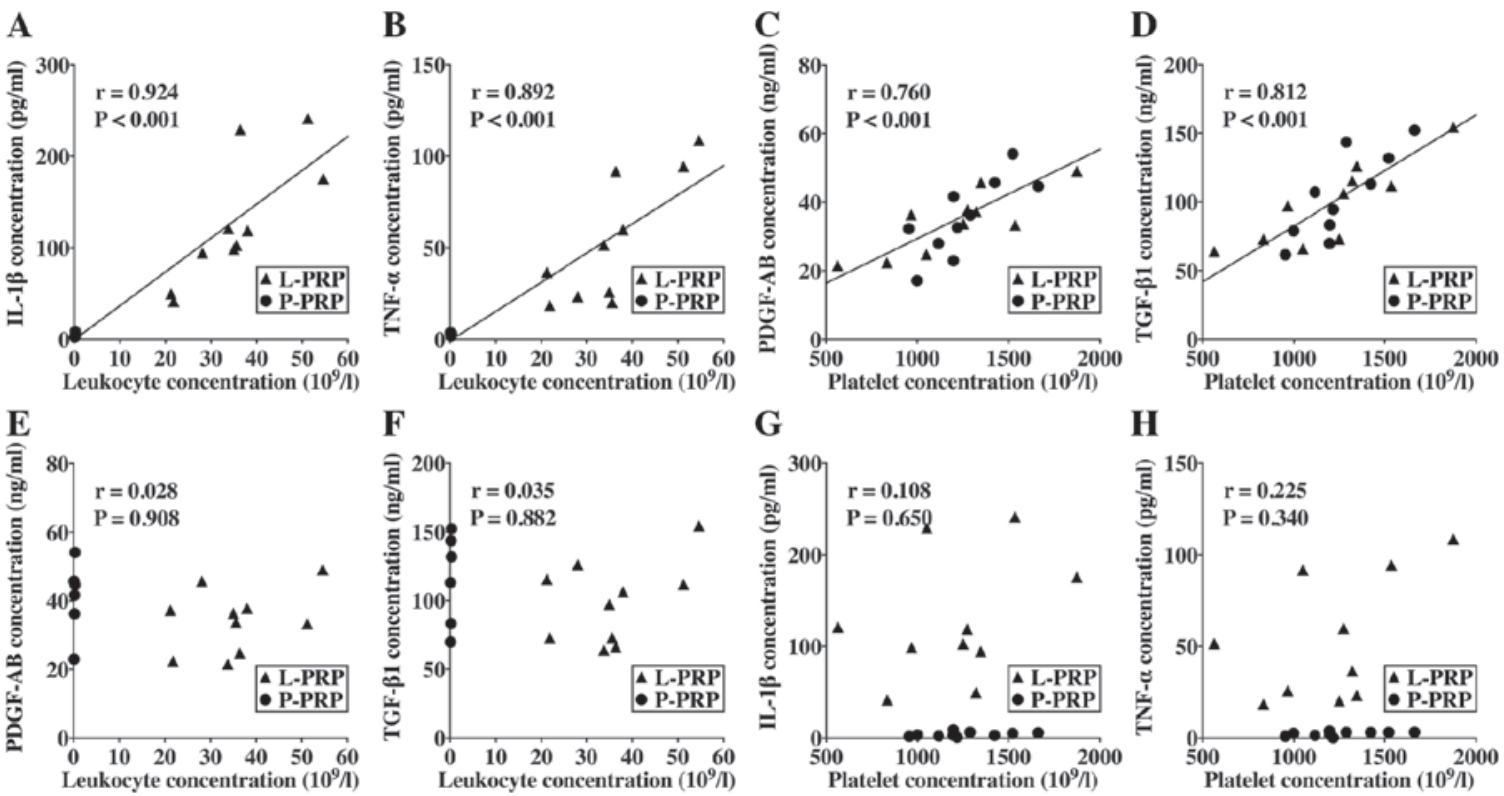

Figure 3. Correlations between components of PRP formulations. There were significant positive correlations between leukocyte and (A) IL-1 $\beta$ concentration, between leukocyte and (B) TNF- $\alpha$ concentration, between platelet and (C) PDGF-AB concentration and between platelet and (D) TGF- $\beta 1$ concentration. No significant correlations were found between leukocyte concentrations and (E) PDGF-AB or (F) TGF- $\beta 1$ concentrations, or between platelet concentrations and (G) IL-1 $\beta$ or (H) TNF- $\alpha$ concentrations. L-PRP, leukocyte and platelet-rich plasma; P-PRP, pure platelet-rich plasma; TNF- $\alpha$, tumor necrosis factor- $\alpha$; TGF- $\beta 1$, transforming growth factor- $\beta 1$; IL- $1 \beta$, interleukin $1 \beta$; PDGF-AB, platelet-derived growth factor-AB.

NO were observed among HACs in the control, PDTC and P-PRP groups. These results indicated that L-PRP may have a pro-inflammatory effect on HACs via activation of the NF- $\mathrm{\kappa B}$ signaling pathway.

Effects of L-PRP and P-PRP on extracellular matrix formation. Western blot analysis revealed that the expression levels of Col II, SOX9 and aggrecan in the cells of the PDTC group and P-PRP group were similar, but were higher, compared with those in the L-PRP group, which, in turn, were higher than those in the control group (Fig. 5A).

A similar trend was observed in the results of the RT-qPCR analysis. The cells in the L-PRP group had higher mRNA expression levels of $\mathrm{Col}$ II ( $\mathrm{P}<0.001$; Fig. 5B), SOX9 ( $\mathrm{P}=0.001$; 


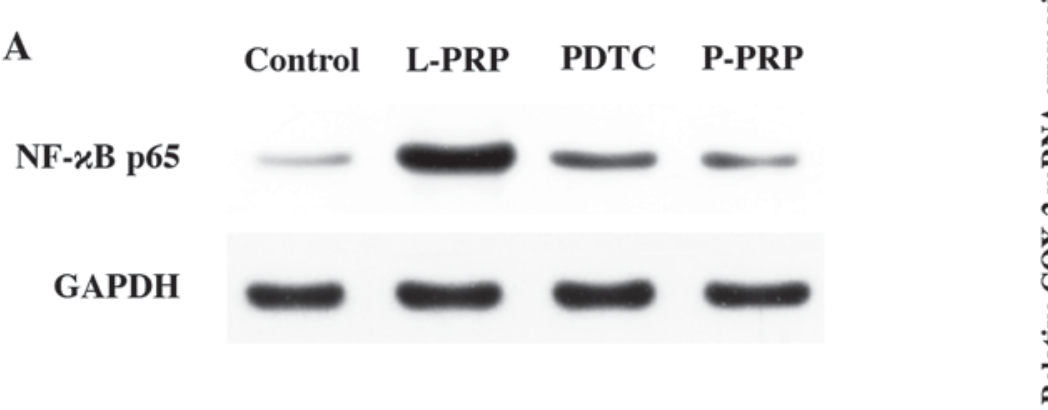

C

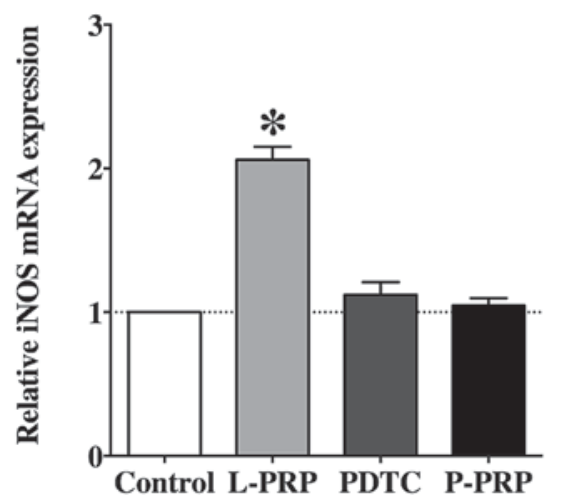

D

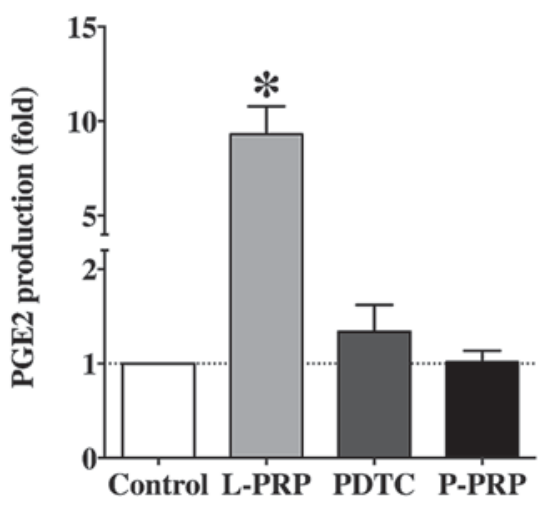

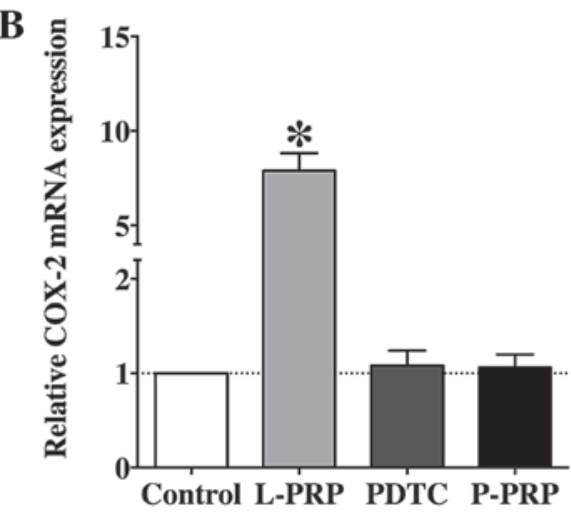

E

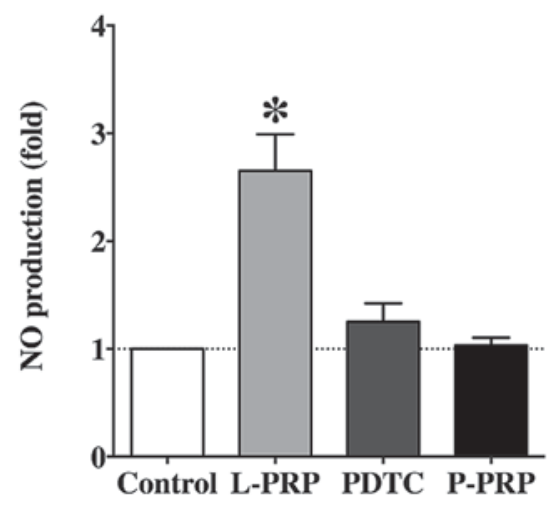

Figure 4. Effects of PRP formulations on the NF- $\kappa$ B signaling pathway. (A) Western blot analysis was performed to analyze the expression of NF- $\mathrm{B}$ p65 in the nucleus. Reverse transcription-quantitative polymerase chain reaction analysis was performed to detect mRNA expression levels of (B) COX-2 and (C) iNOS. The enzyme-linked immunosorbent assay and Griess reaction were performed to determine (D) PGE2 and (E) NO production, respectively. Data were normalized according to the control group. Data are presented as the mean \pm standard deviation. ${ }^{*} \mathrm{P}<0.05$, compared with the control group. L-PRP, leukocyte and platelet-rich plasma; P-PRP, pure platelet-rich plasma; NF- $\mathrm{B}, \mathrm{NF}$, nuclear factor- $\kappa \mathrm{B}$; PDTC, pyrrolidine dithiocarbamate; COX-2, cyclooxygenase-2; iNOS, inducible nitric oxide synthase; PGE2, prostaglandin E2; NO, nitric oxide.

Fig. 5C) and aggrecan ( $\mathrm{P}=0.035$; Fig. 5D), compared with those in the control group. The cells in the PDTC and P-PRP groups had similar, but higher mRNA expression levels of all cartilaginous markers, compared with cells in the L-PRP group $(\mathrm{P}<0.001)$.

In terms of the production of GAG, the cells in the PDTC and P-PRP groups secreted significantly higher levels of GAG, compared with cells in the L-PRP group $(\mathrm{P}=0.015 ; \mathrm{P}=0.012$, respectively), which, in turn, secreted significantly higher levels of GAG, compared with the control group $(\mathrm{P}=0.030$; Fig. 5E). No significant difference was observed in the production of GAG between cells of the PDTC group and P-PRP group $(\mathrm{P}>0.999)$.

These results demonstrated that L-PRP and P-PRP induced distinct effects on the extracellular matrix formation of HACs, with P-PRP exhibiting more marked effects. In addition, inhibiting the activation of $N F-\kappa B$ enhanced the effects of L-PRP to a level similar to that of P-PRP. These findings indicated that the distinct effects of L-PRP and P-PRP on the NF- $\kappa \mathrm{B}$ signaling pathway may be the mechanism underlying the distinct effects of L-PRP and P-PRP on HAC extracellular matrix formation.

\section{Discussion}

The platelets and growth factors, which have been detected consistently in PRP, are considered the basis of PRP therapy for cartilage regeneration. Thus, the present study quantified the platelet and growth factor concentrations to characterize L-PRP and P-PRP. PDGF-AB, which is the primary isoform of human PDGF and binds to $\alpha$ and $\beta$ receptors, is a potent chemokine, and regulates cell proliferation and extracellular matrix deposition (22). TGF- $\beta 1$ has been previously shown to be involved in promoting chondrocyte anabolism in vitro (23). In addition, in vivo injections of these growth factors lead to increased cartilage formation (24). Although other growth factors found in PRP, including IGF, FGF and EGF, also have beneficial effects on the proliferation or extracellular matrix formation of chondrocytes, they have been found to have more variable concentrations in PRP (25) and, thus, were excluded from the assays in the present study. The results showed that the L-PRP and P-PRP used in the present study concentrated platelets and, therefore, PDGF-AB and TGF- $\beta 1$, compared with the whole blood. These findings were in accordance with reported previously (10). Additionally, the present study found that higher growth factor concentrations of PRP formulations were significantly correlated with higher platelet concentrations, but not higher leukocyte concentrations, in the PRP formulations. These findings indicated that it was platelets, rather than leukocytes, that were the major sources of growth factors in PRP formulations.

Of note, although L-PRP had similar platelet and growth factor concentrations to P-PRP, it was not as effective as P-PRP for the promotion of extracellular matrix formation in HACs. 
A

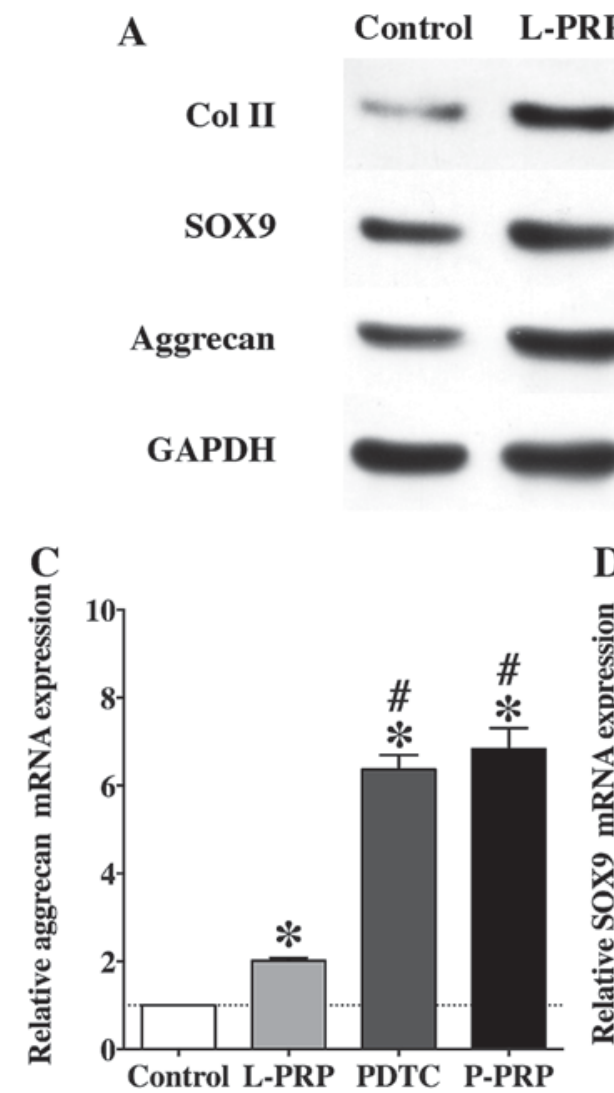

PDTC P-PRP
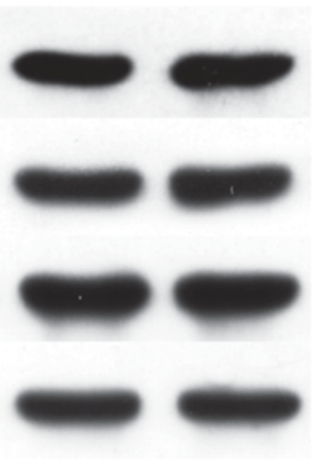

D

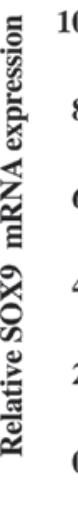

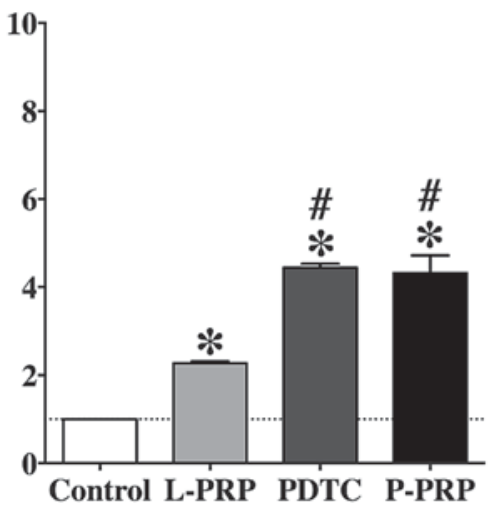

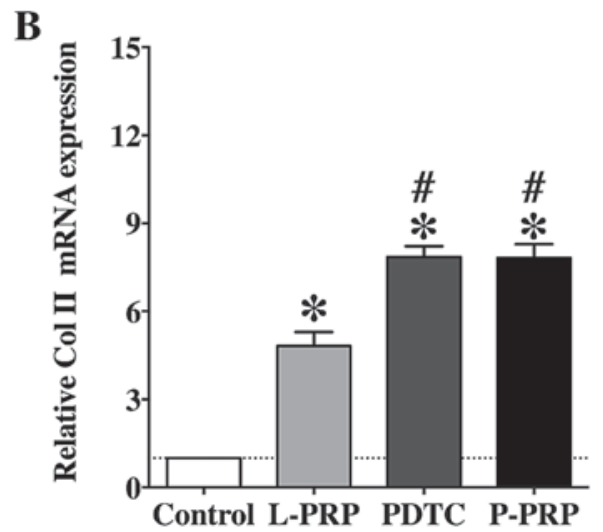

E

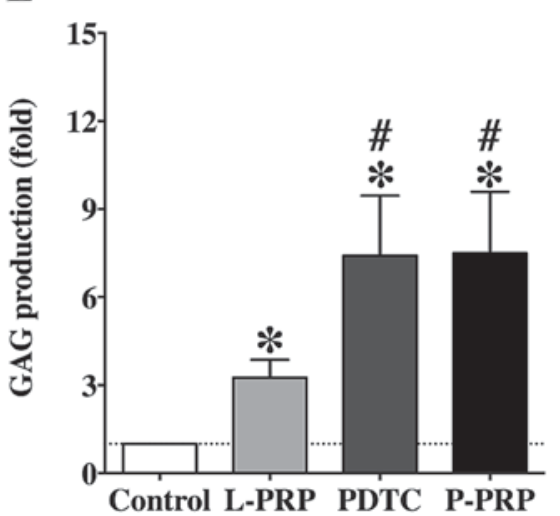

Figure 5. Effects of PRP formulations on extracellular matrix formation. (A) Western blot analysis was performed to analyze the expression of cartilaginous markers. Reverse transcription-quantitative polymerase chain reaction analysis was performed to detect mRNA expression levels of (B) Col II, (C) aggrecan and (D) SOX9. Data were normalized according to the control group. (E) 1,9-dimethylmethylene blue assay was performed to analyze GAG production. Data are presented as the mean \pm standard deviation. "P<0.05, compared with the control group; ${ }^{*} \mathrm{P}<0.05$, compared with the L-PRP group. L-PRP, leukocyte and platelet-rich plasma; P-PRP, pure platelet-rich plasma; PDTC, pyrrolidine dithiocarbamate; Col II, collagen II; SOX9, sex-determining region-box Y; GAG, glycosaminoglycan.

The possible reason for this may be that L-PRP not only concentrated growth factors, which had beneficial effects on the extracellular matrix formation of HACs, but also concentrated IL-1 $\beta$ and TNF- $\alpha$, which are known to be involved in the regulation of inflammatory responses. These findings support those of other studies demonstrating increased levels of IL-1 $\beta$ and TNF- $\alpha$ in L-PRP, and the positive correlations between leukocyte concentration and these pro-inflammatory cytokines in PRP formulations (10). It has been shown in several studies that chondrocytes treated with IL- $1 \beta$ or TNF- $\alpha$ not only demonstrate increased expression of catabolic molecules, including COX-2, iNOS, matrix metalloproteinases and members of the a disintegrin and metalloproteinase with thrombospondin type 1 motifs family (14), but also demonstrate inhibited extracellular matrix component synthesis (26). In addition to acting independently, IL-1 $\beta$ and TNF- $\alpha$ act in synergy to induce articular cartilage destruction in vivo (27). Furthermore, antagonists of IL-1 $\beta$ or TNF- $\alpha$ have been shown to be effective in relieving the symptoms of osteoarthritis $(28,29)$. These findings suggested that the concentrated leukocytes in L-PRP may release increased levels of pro-inflammatory cytokines to counter the beneficial effects of growth factors on the extracellular matrix formation of HACs. This results in the observed inferior effects of L-PRP, compared with P-PRP.

The results of the present study demonstrated that L-PRP resulted in NF- $\kappa \mathrm{B}$ activation, unlike P-PRP. To the best of our knowledge, the present study is the first to demonstrate this.
Several lines of evidence suggest that the NF- $\kappa \mathrm{B}$ signaling pathway is intimately involved in the disturbed metabolism and enhanced catabolism of articular cartilage, which is induced by IL-1 $\beta$ and TNF- $\alpha$ (30). The effects on the NF- $\kappa$ B signaling pathway of PRP formulations, which increase or decrease levels of IL-1 $\beta$ and TNF- $\alpha$, have not been evaluated previously. Although platelet lysate and PRP clot releasate have been shown to inhibit activation of the NF- $\mathrm{NB}$ signaling pathway $(31,32)$, these products contain no viable leukocytes, which are concentrated in L-PRP and may be the major sources of pro-inflammatory cytokines or viable platelets, which are also concentrated in L-PRP, and P-PRP and may be the major sources of growth factors. In addition, an excess of leukocytes can overwhelm the ability of growth factors to modulate the effects of pro-inflammatory cytokines, which is considered to be the basis of the effects of platelet lysate and PRP clot releasate on the NF- $\kappa \mathrm{B}$ signaling pathway $(33,34)$. Therefore, platelet lysate and PRP clot releasate have distinct characteristics, compared with the PRP formulations used in clinical practice, and thus, the effects of these products on the $\mathrm{NF}-\kappa \mathrm{B}$ signaling pathway may not reflect that of PRP in clinical practice.

The distinct effects of L-PRP and P-PRP on the NF- $\kappa \mathrm{B}$ signaling pathway may be the mechanism underlying the distinct effects of L-PRP and P-PRP on HAC extracellular matrix formation. The findings of the present study demonstrated that PDTC, an inhibitor of NF- $\kappa$ B activation, which suppressed the 
effects of L-PRP on the NF- $\mathrm{B}$ signaling pathway, enhanced the effects of L-PRP on HAC extracellular matrix formation to a level similar to that of P-PRP. Therefore, activation of the $\mathrm{NF}-\kappa \mathrm{B}$ signaling pathway may counter the beneficial effects of growth factors on extracellular matrix formation and result in the inferior effects of L-PRP, compared with P-PRP. The NF- $\mathrm{B}$ signaling pathway is also involved in pain (35), and inhibition of the activation of NF- $\kappa \mathrm{B}$ has been advocated as a novel drug target for pain relief (36). Therefore, $N F-\kappa B$ activation may be involved in the increased incidence of pain reaction following L-PRP injections, which is observed in clinical practice. Further investigations are required to evaluate this in vivo.

In conclusion, although L-PRP promoted the extracellular matrix formation of HACs, leukocytes in L-PRP may activate the $N F-\kappa B$ signaling pathway via the delivery of IL- $1 \beta$ and TNF- $\alpha$ to counter the beneficial effects of growth factors on HAC extracellular matrix formation, leading to inferior effects, compared with P-PRP. Thus, P-PRP may be more suitable for the treatment of osteoarthritis. Further investigations are required to evaluate these findings in vivo to identify a PRP formation, which is more suitable for the treatment of osteoarthritis in clinical practice.

\section{Acknowledgements}

This study was supported by a grant from the National Natural Science Foundation of China (grant no. 81401799) and a Shanghai Youth Start-up grant (grant no. 14YF1412100).

\section{References}

1. Woolf AD and Pfleger B: Burden of major musculoskeletal conditions. Bull World Health Organ 81: 646-656, 2003.

2. FitzGerald GA and Patrono C: The coxibs, selective inhibitors of cyclooxygenase-2. N Engl J Med 345: 433-442, 2001.

3. Altman RD and Moskowitz R: Intraarticular sodium hyaluronate (Hyalgan) in the treatment of patients with osteoarthritis of the knee: a randomized clinical trial. Hyalgan Study Group. J Rheumatol 25: 2203-2212, 1998.

4. Muir H: The chondrocyte, architect of cartilage. Biomechanics, structure, function and molecular biology of cartilage matrix macromolecules. Bioessays 17: 1039-1048, 1995.

5. Castillo TN, Pouliot MA, Kim HJ and Dragoo JL: Comparison of growth factor and platelet concentration from commercial platelet-rich plasma separation systems. Am J Sports Med 39: 266-271, 2011.

6. Magalon J, Bausset O, Serratrice N, Giraudo L, Aboudou H, Veran J, Magalon G, Dignat-Georges F and Sabatier F: Characterization and comparison of 5 platelet-rich plasma preparations in a single-donor model. Arthroscopy 30: 629-638, 2014.

7. Kon E, Mandelbaum B, Buda R, Filardo G, Delcogliano M, Timoncini A, Fornasari PM, Giannini S and Marcacci M: Platelet-rich plasma intra-articular injection versus hyaluronic acid viscosupplementation as treatments for cartilage pathology: From early degeneration to osteoarthritis. Arthroscopy 27: 1490-1501, 2011

8. Filardo G, Kon E, Buda R, Timoncini A, Di Martino A, Cenacchi A, Fornasari PM, Giannini S and Marcacci M Platelet-rich plasma intra-articular knee injections for the treatment of degenerative cartilage lesions and osteoarthritis. Knee Surg Sports Traumatol Arthrosc 19: 528-535, 2011.

9. Dohan Ehrenfest DM, Rasmusson L and Albrektsson T: Classification of platelet concentrates: From pure platelet-rich plasma (P-PRP) to leucocyte- and platelet-rich fibrin (L-PRF). Trends Biotechnol 27: 158-167, 2009.

10. Sundman EA, Cole BJ and Fortier LA: Growth factor and catabolic cytokine concentrations are influenced by the cellular composition of platelet-rich plasma. Am J Sports Med 39: 2135-2140, 2011.
11. Burguera EF, Vela-Anero A, Magalhaes J, Meijide-Failde R and Blanco FJ: Effect of hydrogen sulfide sources on inflammation and catabolic markers on interleukin $1 \beta$-stimulated human articular chondrocytes. Osteoarthritis Cartilage 22: 1026-1035, 2014.

12. Campbell KA, Minashima T, Zhang Y, Hadley S, Lee YJ, Giovinazzo J, Quirno M and Kirsch T: Annexin A6 interacts with p65 and stimulates NF-kB activity and catabolic events in articular chondrocytes. Arthritis Rheum 65: 3120-3129, 2013.

13. Altman R, Asch E, Bloch D, Bole G, Borenstein D, Brandt K, Christy W, Cooke TD, Greenwald R, Hochberg M, et al: Development of criteria for the classification and reporting of osteoarthritis. Classification of osteoarthritis of the knee. Diagnostic and therapeutic criteria committee of the American rheumatism association. Arthritis Rheum 29: 1039-1049, 1986.

14. Cavallo C, Filardo G, Mariani E, Kon E, Marcacci M, Pereira Ruiz MT, Facchini A and Grigolo B: Comparison of platelet-rich plasma formulations for cartilage healing: An in vitro study. J Bone Joint Surg Am 96: 423-429, 2014.

15. Zhu H, Cheng X, Niu X, Zhang Y, Guan J, Liu X, Tao S, Wang Y and Zhang C: Proton-sensing GPCR-YAP signalling promotes cell proliferation and survival. Int J Biol Sci 11: 1181-1189, 2015.

16. Zhi Y, Duan Y, Zhou X, Yin X, Guan G, Zhang H, Dong Q and Yang K: NF-kB signaling pathway confers neuroblastoma cells migration and invasion ability via the regulation of CXCR4. Med Sci Monit 20: 2746-2752, 2014.

17. Mladenova D, Pangon L, Currey N, Ng I, Musgrove EA, Grey ST and Kohonen-Corish MR: Sulindac activates NF-kB signaling in colon cancer cells. Cell Commun Signal 11: 73, 2013.

18. de Mos M, van der Windt AE, Jahr H, van Schie HT, Weinans H, Verhaar JA and van Osch GJ: Can platelet-rich plasma enhance tendon repair? A cell culture study. Am J Sports Med 36: 1171-1178, 2008.

19. McCarrel T and Fortier L: Temporal growth factor release from platelet-rich plasma, trehalose lyophilized platelets, and bone marrow aspirate and their effect on tendon and ligament gene expression. J Orthop Res 27: 1033-1042, 2009.

20. Xie X, Wang Y, Zhao C, Guo S, Liu S, Jia W, Tuan RS and Zhang C: Comparative evaluation of MSCs from bone marrow and adipose tissue seeded in PRP-derived scaffold for cartilage regeneration. Biomaterials 33: 7008-7018, 2012.

21. Livak KJ and Schmittgen TD: Analysis of relative gene expression data using real-time quantitative PCR and the 2(-Delta Delta C(T)) Method. Methods 25: 402-408, 2001.

22. Heldin $\mathrm{CH}$, Eriksson U and Ostman A: New members of the platelet-derived growth factor family of mitogens. Arch Biochem Biophys 398: 284-290, 2002.

23. Yaeger PC, Masi TL, de Ortiz JL, Binette F, Tubo R and McPherson JM: Synergistic action of transforming growth factor-beta and insulin-like growth factor-I induces expression of type II collagen and aggrecan genes in adult human articular chondrocytes. Exp Cell Res 237: 318-325, 1997.

24. Stewart K, Pabbruwe M, Dickinson S, Sims T, Hollander AP and Chaudhuri JB: The effect of growth factor treatment on meniscal chondrocyte proliferation and differentiation on polyglycolic acid scaffolds. Tissue Eng 13: 271-280, 2007.

25. Berg U, Gustafsson T, Sundberg CJ, Carlsson-Skwirut C, Hall K, Jakeman P and Bang P: Local changes in the insulin-like growth factor system in human skeletal muscle assessed by microdialysis and arterio-venous differences technique. Growth Horm IGF Res 16: 217-223, 2006.

26. Saklatvala J: Tumour necrosis factor alpha stimulates resorption and inhibits synthesis of proteoglycan in cartilage. Nature 322: 547-549, 1986.

27. Henderson B and Pettipher ER: Arthritogenic actions of recombinant IL-1 and tumour necrosis factor alpha in the rabbit: Evidence for synergistic interactions between cytokines in vivo. Clin Exp Immunol 75: 306-310, 1989.

28. Bacconnier L, Jorgensen C and Fabre S: Erosive osteoarthritis of the hand: Clinical experience with anakinra. Ann Rheum Dis 68: 1078-1079, 2009.

29. Grunke M and Schulze-Koops H: Successful treatment of inflammatory knee osteoarthritis with tumour necrosis factor blockade. Ann Rheum Dis 65: 555-556, 2006.

30. Davidson RK, Jupp O, de Ferrars R, Kay CD, Culley KL, Norton R, Driscoll C, Vincent TL, Donell ST, Bao Y and Clark IM: Sulforaphane represses matrix-degrading proteases and protects cartilage from destruction in vitro and in vivo. Arthritis Rheum 65: 3130-3140, 2013. 
31. Pereira RC, Scaranari M, Benelli R, Strada P, Reis RL, Cancedda R and Gentili C: Dual effect of platelet lysate on human articular cartilage: A maintenance of chondrogenic potential and a transient proinflammatory activity followed by an inflammation resolution. Tissue Eng Part A 19: 1476-1488, 2013

32. van Buul GM, Koevoet WL, Kops N, Bos PK, Verhaar JA, Weinans H, Bernsen MR and van Osch GJ: Platelet-rich plasma releasate inhibits inflammatory processes in osteoarthritic chondrocytes. Am J Sports Med 39: 2362-2370, 2011.

33. Pujol JP, Chadjichristos C, Legendre F, Bauge C, Beauchef G, Andriamanalijaona R, Galera P and Boumediene K: Interleukin-1 and transforming growth factor-beta 1 as crucial factors in osteoarthritic cartilage metabolism. Connect Tissue Res 49: 293-297, 2008 .
34. Rédini F, Mauviel A, Pronost S, Loyau G and Pujol JP: Transforming growth factor beta exerts opposite effects from interleukin-1 beta on cultured rabbit articular chondrocytes through reduction of interleukin-1 receptor expression. Arthritis Rheum 36: 44-50, 1993.

35. Millan MJ: The induction of pain: An integrative review. Prog Neurobiol 57: 1-164, 1999.

36. Tegeder I, Niederberger E, Schmidt R, Kunz S, Guhring H, Ritzeler O, Michaelis M and Geisslinger G: Specific Inhibition of IkappaB kinase reduces hyperalgesia in inflammatory and neuropathic pain models in rats. J Neurosci 24: 1637-1645, 2004. 